\title{
Consent for blood transfusion: summary of recommendations from the Advisory Committee for the Safety of Blood, Tissues and Organs (SaBTO)
}

\author{
Authors: Michael F Murphy, ${ }^{A}$ Andrea Harris ${ }^{B}$ and James Neuberger; ${ }^{C}$ on behalf of the SaBTO Consent for \\ Transfusion Working Group
}

The Advisory Committee on the Safety of Blood, Tissues and Organs (SaBTO) decided that its 2011 recommendations on consent for blood transfusion needed to be reviewed and revised due to evidence of poor compliance and recent legal guidance on consent. The recommendations are to ensure that patients are informed about and understand the purpose, benefits and potential risks of transfusion, and have an opportunity to discuss their treatment options. They should be incorporated into local practices for all patients.

KEYWORDS: consent, blood transfusion, recommendations, transfusion safety

DOI: $10.7861 /$ clinmed.2020-1035

\section{Introduction}

Patients should be fully informed of the reasons for blood transfusion, its benefits, risks and alternatives, and give their consent. The Advisory Committee on the Safety of Blood, Tissues and Organs (SaBTO) decided that its 2011 recommendations needed to be reviewed and revised due to evidence of poor compliance and recent legal guidance on consent. ${ }^{1-3}$ The objectives were to enhance standards and good practice for the provision of information about blood transfusion and for obtaining patient consent.

\section{Background}

Blood transfusion is common in clinical practice. Blood transfusion is well known to be associated with adverse effects, and surveys of the use of blood in the UK indicate $20 \%$ or more of transfusions are inappropriate and that alternatives to transfusion are underused. ${ }^{4,5}$

Authors: ${ }^{\text {A }}$ professor of transfusion medicine and consultant haematologist, NHS Blood and Transplant and Oxford NIHR Biomedical Research Centre, Oxford, UK; ${ }^{\text {B }}$ Clinical services professional nursing lead and chair of Consent for Transfusion Working Group, NHS Blood and Transplant, UK; ${ }^{C}$ professor of medicine, consultant transplant physician and chair of SaBTO, NHS Blood and Transplant and University of Birmingham, Birmingham, UK
It is therefore essential that patients are fully informed of the benefits, risks and alternatives to transfusion, and give their consent. SaBTO is the independent advisory committee that advises ministers of the UK nations on the safety of blood, tissues and organs. In 2011, SaBTO made recommendations on patient consent for blood transfusion. In 2014, the National Comparative Audit of Consent for Blood Transfusion found that the implementation of the SaBTO recommendations was sporadic and compliance was generally low. ${ }^{2}$ Since 2011, the UK Supreme Court Montgomery $\checkmark$ Lanarkshire ruling provided additional guidance on consent, and the ongoing Infected Blood Inquiry has identified concerns about whether and to what extent people were treated without knowledge or consent. ${ }^{3,6}$

In view of these developments, SaBTO decided that the recommendations needed to be reviewed and revised, as necessary, to enhance standards for the provision of information about blood transfusion and for obtaining patient consent and clarify good practice (Boxes 1 and 2).

Considerations leading to the development of the recommendations

> The decision of the UK Supreme Court in Montgomery v Lanarkshire was a landmark legal decision for informed consent. ${ }^{3}$ The Supreme Court held that a patient should be told whatever they want to know, not what the doctor thinks they should be told, and established a duty of care to warn of material risks. The test of materiality defined in the

Box 1. How patients were involved in the development of the recommendations

Two of the members of SaBTO's working group on consent for blood transfusion were lay representatives: one is a lay representative on SaBTO and the other is a member of the National Blood Transfusion Committee's Patient Involvement Working Group.

The following patient groups were consulted: Leukaemia Care; Myeloma UK; MDS UK; National Blood Transfusion Committee Patient Involvement Working Group; Royal College of Anaesthetists Lay Committee; Thalassaemia Society; The Patients Association; and the Sickle Cell Society. 
Box 2. How feedback was sought and incorporated into the recommendations

In addition to the involvement of patients and patient support groups cited in Box 1, there were widespread consultations with interested parties and stakeholders. Responses to the consultation were received from 50 acute NHS trusts, seven royal colleges, the UK Blood Services, the British Society for Haematology, the British Orthopaedic Association, the British Medical Association and Serious Hazards of Transfusion (SHOT). Legal advice was sought from the legal representatives of all fou UK nations.

All responses were scrutinised by the chair of the SaBTO Consent for Blood Transfusion Working Group, and the chair of SaBTO, with oversight from other working group members, and some changes to the recommendations were made.

Montgomery ruling was whether 'a reasonable person in the patient's circumstances would be likely to attach significance to the risk, or the doctor is or should reasonably be aware that the particular patient would be likely to attach significance to it'. It represents a shift towards a more collaborative approach to consent between patients and health practitioners, and means finding the time to explain the risks and benefits of a recommended course of action and the other options.

> Other guidance, guidelines and recommendations which were taken into account were the General Medical Council (GMC) guidance Good Medical Practice; updated UK variant CreutzfeldtJakob (vCJD) precautionary measures; the 2015 National Institute for Health and Care Excellence (NICE) blood transfusion guideline; the 2016 NICE Blood Transfusion Quality Standards; and the 2015 Choosing Wisely recommendations for blood transfusion. ${ }^{7-11}$

$>$ Blood transfusion for the purposes of these recommendations refers to the transfusion of blood components, as defined by the Blood Safety and Quality Regulations (BSQR SI 2005 No 50 as amended) which defines blood components as a therapeutic constituent of blood (red blood cells, platelets, fresh-frozen plasma (FFP), cryoprecipitate and granulocytes). ${ }^{12}$ Blood products (such as albumin or intravenous immunoglobulin) are generally out of scope as these are classified as medicinal products and subject to different regulations.

> The recommendations are pertinent to all patients who may be exposed to blood components (therefore including, for example, patients undergoing extracorporeal membrane oxygenation (ECMO), pump priming or organ perfusion) and to both autologous (obtained from the same individual) and allogeneic (donated) transfusions as many of the most frequent serious risks of transfusion (eg transfusion associated circulatory overload (TACO) and wrong blood component transfused) are similar. ${ }^{4}$

$>$ It is not the purpose of the recommendations to provide detailed guidance related to paediatrics, reduced mental capacity or refusal of blood components (including advanced directives), or to advise on legalities related to consent, which should be covered by standard hospital practices.

\section{Recommendations}

The recommendations are to ensure that patients are informed about and understand the purpose, benefits and potential risks of transfusion, and have an opportunity to discuss their treatment options. They are a set of principles which should be incorporated into local practices for all patients, taking into account specific issues related to paediatric patients and those with deemed mental incapacity.

> Informed and valid consent for transfusion is obtained for all patients who will likely, or definitely, receive a transfusion, for example, where blood is routinely requested prior to surgery or where a 'group and save' or 'cross-match' sample is taken pre-procedure.

> Consideration should be given whether the transfusion is the only available treatment, whether any alternative treatments are available and suitable, and the risks and benefits of those alternatives to transfusion.

> The duration of consent needs to be discussed and agreed with the patient as part of the shared decision-making process. It is recognised that there is a difference between a patient who receives regular transfusions every few weeks for that condition (eg, with haemoglobinopathy) and a patient with cancer who has surgery, then a course of chemotherapy and then further surgery, with each treatment stage potentially requiring transfusion. If it is deemed appropriate that consent may span more than one transfusion episode, or across the duration of a patient admission period, this should be documented in the patient's clinical notes.

$>$ In addition to the provision of information about the nature and purpose of the proposed treatment, an active discussion should result in shared decision making, allowing the patient to ask their own questions, and to raise any concerns that they wish to be addressed before they make a decision to receive, or refuse, the transfusion.

> Patients who have a blood transfusion and who were not able to give informed and valid consent prior to the transfusion are informed of the transfusion prior to discharge and provided with relevant information either in paper or electronic format.

> All patients who have received a transfusion should be provided with details of the transfusion (type(s) of component), together with information about any adverse events associated with the transfusion. Patients should also be informed that they are no longer eligible to donate blood. All of this information should be included in their hospital discharge summary to ensure their family doctor is also aware.

> The UK Blood Services provide a standardised source of information for patients who may receive a blood transfusion in the UK.

> Training in consent for transfusion is included in all relevant undergraduate healthcare practitioners training, followed by continuous, regular knowledge updates (minimum 3-yearly) for all healthcare practitioners involved in the consent for transfusion process.

> There is a centralised UK wide information resource for healthcare practitioners to facilitate consent for transfusion discussions, indicating the key issues to be discussed when obtaining informed and valid consent for a blood transfusion, and providing up-to-date information on the risks of transfusion. This resource should be provided by the UK blood services.

$>$ All UK healthcare organisations who provide blood transfusions employ mechanisms (such as audit) to monitor the implementation and compliance with these SaBTO 
recommendations, with subsequent improvement plans developed and implemented if indicated.

\section{Key points}

> Patients should be informed about and understand the purpose, benefits and potential risks of transfusion, and have an opportunity to discuss their treatment options.

> The information provided should include whether the transfusion is the only available treatment, whether any alternative treatments are available and suitable, and the risks and benefits of those alternatives to transfusion.

> The amount of information required to make consent truly informed may vary depending on the complexity and risks of treatment as well as the patient's wishes.

> Consent should be obtained and documented for those who will or might receive (as evidenced by a sending of a specimen for 'group and save' or 'cross-match') a transfusion of blood or components (including red blood cells, platelets, FFP, cryoprecipitate and granulocytes) or being exposed to blood as in, for example, ECMO.

> Where transfusion may be required long term (eg, for those with sickle cell disease or undergoing chemotherapy), written consent needs be obtained only at the start of treatment and at 5-yearly intervals, although consent should be confirmed verbally before each transfusion.

> A standardised source of information should be developed for patients who may receive a blood transfusion in the UK, and training provided for all healthcare practitioners involved in the consent for transfusion process.

\section{Acknowledgements}

The members of the working group were Andrea Harris (chair; nurse), James Neuberger (chair of SaBTO; transplant physician), Charles Baker (anaesthetist), Ann Benton (haematologist), Damien Carson (anaesthetist), Ann Davidson (nurse), Roger Graham (lay member), Mike Murphy (haematologist), Shruthi Narayan (haematologist and medical director, SHOT), Megan Rowley (haematologist) and Rhonda Skeete (lay member).

\section{References}

1 Advisory Committee on the Safety of Blood, Tissues and Organs. Patient consent for blood transfusion. Department of Health and Social Care, 2011. www.gov.uk/government/publications/patientconsent-for-blood-transfusion

2 NHS Blood and Transplant. National Comparative Audit of Blood Transfusion: 2014 audit of patient information \& consent. NHS Blood and Transplant, 2014. https://nhsbtdbe.blob.core.windows. net/umbraco-assets-corp/14925/2014-audit-of-patient-information-consent.pdf

3 Montgomery (Appellant) v Lanarkshire Health Board (Respondent) (Scotland). [2015] UKSC 11. www.supremecourt.uk/cases/uksc2013-0136.htm

4 Serious Hazards of Transfusion. Annual SHOT Report 2019. SHOT, 2020. www.shotuk.org/wp-content/uploads/myimages/SHOTREPORT-2019-Final-Bookmarked-v2.pdf

5 NHS Blood and Transplant. National comparative audit of blood transfusion. NHS, 2020. https://hospital.blood.co.uk/audits/ national-comparative-audit

6 Infected Blood Inquiry. www.infectedbloodinquiry.org.uk

7 General Medical Council. Good Medical Practice. GMC, 2020. www. gmc-uk.org/ethical-guidance/ethical-guidance-for-doctors/goodmedical-practice

8 Department of Health and Social Care. Minimise transmission risk of CJD and VCJD in healthcare settings. GOV.UK, 2017. www. gov.uk/government/publications/guidance-from-the-acdp-tse-riskmanagement-subgroup-formerly-tse-working-group

9 National Institute for Health and Care Excellence. Blood transfusion: NICE guideline [NG24]. NICE, 2015. www.nice.org.uk/guidance/ng24

10 National Institute for Health and Care Excellence. Blood transfusion: Quality standard [QS138]. NICE, 2016. www.nice.org.uk/guidance/ qs138

11 Choosing Wisely UK. www.choosingwisely.co.uk

12 The Blood and Safety Quality Regulations 2005. UK Parliament, 2005. www.legislation.gov.uk/uksi/2005/50/contents/made.

Address for correspondence: Prof Michael F Murphy, NHS Blood and Transplant, John Radcliffe Hospital, Headley Way, Headington, Oxford OX3 9BQ, UK.

Email: mike.murphy@nhsbt.nhs.uk

Twitter: @MurphyProf 